\title{
Image analysis of hydrophobicity of polymer insulators using PVM.
}

\author{
Tetsuro Tokoro, Yûki Omoto and Masamitsu Kosaki \\ Gifu National College of Technology, Motosu, Gifu, Japan.
}

\begin{abstract}
Hydrophobicity of polymeric insulating material surface such as silicone rubber insulator (SIR) was studied by using image data analysis of the sample surface. Using PVM (Parallel Virtual Machine) programming procedure for UNIX operating system, hydrophobicity of polymer surface was evaluated from their surface mages of water droplets on the sample surface. The hydrophobic surface images under the spraying of distilled water were taken by CCD camcorder The video image was divided into each image frame and PVM program analyzed each image data, simultaneously.

Image indexes such as size and shape factor, fc, of droplets can evaluate from each hydrophobic image frame. Then, the distribution of the size and fc were evaluated. Time variation of the hydrophobic indexes during high electric field application was also evaluated, where electric field deformed the droplets.
\end{abstract}

\section{Introduction}

Polymeric insulating materials such as SIR are widely used in the manufacture of weather-sheds of outdoor insulators. Silicone rubber especially has excellent hydrophobic property in addition to its excellent electrical, mechanical and chemical characteristics. The usage is expanding rapidly as an electric insulation material typically under the heavy contaminated environment. Therefore, it is very important to study the degradation of hydrophobicity of polymer surface as one of the indexes of the initial deterioration processes.

The hydrophobicity of polymer surface is reduced during the aging; however, it recovers during the rest time in air. It is usually measured by contact angle of a droplet of distilled water on the surface. However, it sometimes shows hysteresis, time dependence and location dependence of the measured point. Therefore, hydrophobic image of the surface is recently used to determine the hydrophobicity class (STRI's HC level from 1 to 7) [1].

A digital image processing method for estimating the level of hydrophobicity of specimens are reported in $[2$, 3]. Relation of STRI indexes from 1 to 7 and image indexes are summarized in Table 1. This paper studies the image data analysis of hydrophobicity by using PVM programming on the UNIX operating system. Image data indexes such as size distribution and shape factor, fc, of the water droplets are evaluated to discuss the HC levels.

Polymer materials sometimes show the time variation of hydrophobic property by absorbing and evaporating the water in the material. Therefore, the evaluation of the dynamic transition of hydrophobic indexes is studied in this paper. To analyze the time variation of the images, extensively amount of image data processing is required and it takes enormous time to calculate. To analyze dynamic transition of the images, PVM was used. It can proceed with the quantitative evaluation of the hydrophobicity by using numbers of computers, simultaneously. Using the time domain changes of the hydrophobic image indexes, PVM also expected to improve the accuracy of the evaluation of image indexes that can indicate the small changes of hydrophobicity class of the sample surface.

Furthermore, this PVM system can diagnosis the vibration of the water droplets on the polymer surface during high ac voltage application. This vibration may initiate the flashover on the insulator surface and, thereafter, the degradation of the material starts.

Table 1: Hydrophobicity class and image indexes.

\begin{tabular}{c|c|c|c}
\hline Hydrophobicity & STRI's HC level & Residual contact angle $(\theta \mathrm{r})$ & Image indexes (fc and size) \\
\hline Hydrophobic & 1 & $\theta \mathrm{r}>80[$ degree $]$ & fc nearly equals 1, very small size \\
& 2 & $80>\theta \mathrm{r}>50$ & fc less than 1, small size \\
& 3 & $50>\theta \mathrm{r}>20$ & fc becomes smaller, larger size \\
\cline { 2 - 4 } & 4,5 & $\theta \mathrm{r}>0$, depends on the location & More small fc, droplets and wet area \\
\cline { 2 - 4 } Hydrophilic & 6,7 & $\theta \mathrm{r}=0$ & $\mathrm{fc}=0$, larger wet area \\
\hline
\end{tabular}




\section{Configuration of PVM Computers}

The configuration of PVM computer system is shown in Figure 1. Master computer controls all slave computers using network system. All computers used in this study are Fujitsu-SUN, GP400S model5. Network speed is 100 Mbps. 50 computers can use in this network, simultaneously. Master computer sends all programs and image data to slave computers and receives each result from slave computers. Number of slave computers used to evaluate the PVM system in this study is from 1 to 3 .

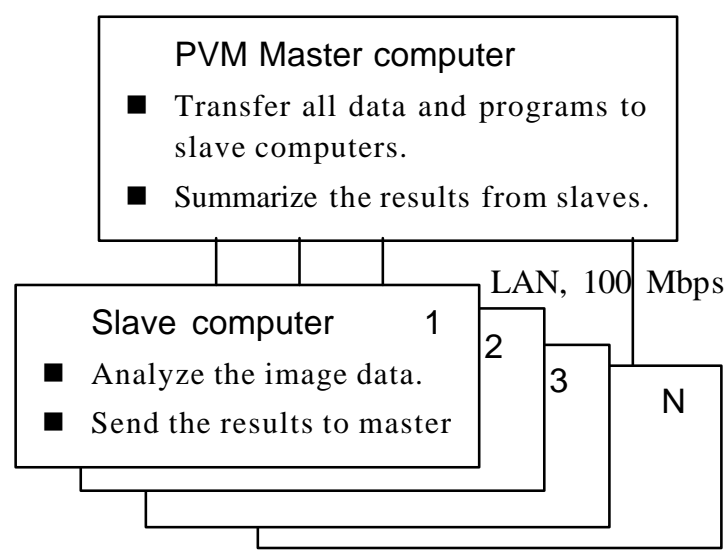

Figure 1: PVM system with master and slave computers.

\section{Performance of PVM System}

The file size of each image data is about 168.56 [kBytes], that is using "pgm" picture format with 430x392 [pixels] and 8bit gray scale. Using $100 \mathrm{Mbps}$ network, the transfer time of each image frame is less than $0.013 \mathrm{sec}$.

Typical image frame used in this study is shown in Figure 2. It has 1503 droplets in the image. Images with 828 and 1146 droplets are also used. The recognizing time of all droplets in each hydrophobic image frame depends on the number of the droplets that has included in the image. Figure 3 shows the CPU time to evaluate all droplets in the image. Increasing the number of water droplets in each image, CPU time also increased. To reduce the total evaluation time of image indexes, only 253 droplets are recognized by PVM slave program in this study. The 253 droplets are recognized from the center of the image toward the edge of the image frame.

If the image has larger number of the droplets, the evaluation time of image indexes slightly increases. The processing time of each image is summarized in Table 2. Even the number of recognized water droplets

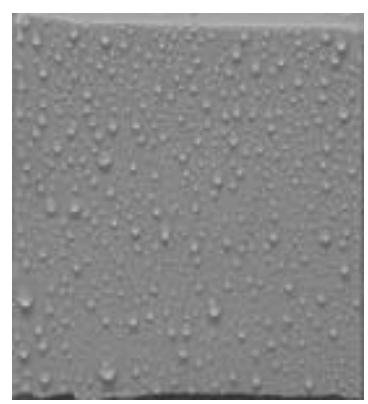

Figure 2: Typical hydrophobic image used in this study. This image has 1503 droplets and requires 42 [s] to analyze.

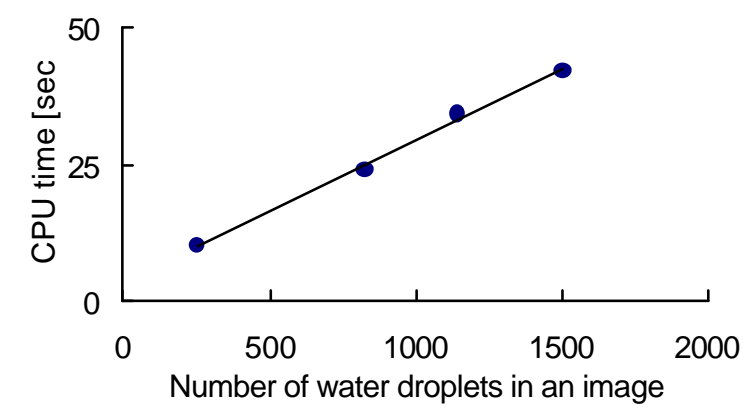

Figure 3: CPU time to evaluate all droplets in an image. Only one slave computer calculates all in each image.

Table 2: CPU processing time for each image.

\begin{tabular}{c|c}
\hline $\begin{array}{c}\text { Procedure of image data to } \\
\text { evaluate the HC levels }\end{array}$ & $\begin{array}{c}\text { CPU time to } \\
\text { calculate } \\
253 \text { droplets }\end{array}$ \\
\hline Transfer time of an image & $0.013[\mathrm{~s}]$ \\
\hline Analyze an image with 828 droplets & $14.7[\mathrm{~s}]$ \\
\hline The one with 1146 droplets & $15.0[\mathrm{~s}]$ \\
\hline The one with 1503 droplets & $16.0[\mathrm{~s}]$ \\
\hline
\end{tabular}

is fixed to 253 , increasing the number of the droplets in the image from 828 to 1503 , the evaluation time also increased slightly from 14.7 to 16.0 [s].

Using PVM image-processing system, the evaluation time of $\mathrm{HC}$ level is reduced drastically. The total time, $\mathrm{T}$, required to analyze all image frames are shown in (1),

$$
T=16.0 \times \frac{N_{I}}{N_{S}}+0.013 \times N_{I}
$$

where, $\mathrm{N}_{\mathrm{I}}$ is the number of the image frames, $\mathrm{N}_{\mathrm{S}}$ is the number of slave computers used simultaneously and 16.0 is the maximum time required for each image. If $\mathrm{N}_{\mathrm{I}}$ is smaller than the maximum number of slave computers, $\mathrm{N}_{\mathrm{S}}$ is set to $\mathrm{N}_{\mathrm{I}}$. 


\section{Digitizing Error of PVM System}

Figure 4 shows the evaluation error of fc, which is calculated by both the PVM and NIH Image program. In this evaluation, circles with diameter, $d$, are made by MS Paint. Shape factor, fc, is calculated by (2),

$$
f_{c}=4 \pi \frac{S}{l^{2}}
$$

where $S$ is a size of circle (droplet) in [pixels], 1 is length of the edge around the circle (droplet) in [pixel]. If the size of the circle is very large, $S=\pi \mathrm{d}^{2} / 4$ and $l=\pi \mathrm{d}$, therefore, $\mathrm{fc}=1(100 \%)$.

Figure 4 shows both image analyzing systems have larger digitizing error when the circle is smaller. Therefore, the capture size of the image of droplets is very important to evaluate the HC levels. Digital image size of water droplet is easy to change by zoom magnitude of CCD camcorder. However, calibration of the image indexes is available.

Table 3 shows digital circle with diameter, $\mathrm{d}=5$, made by MS Paint and its calculated values of S, 1 and fc of both PVM and NIH Image. One of the reasons of this large digitizing error is the difference of the definition of diameter, $d$, of the circle. Assuming the $d=4$ instead of $\mathrm{d}=5$, the digitizing error of both PVM and NIH becomes smaller. The difference of the results by PVM and NIH are mainly caused by the treatment of the edge of image, that is included in a size of droplet (NIH) or not (PVM).

This digitizing error of the calculation of image indexes of PVM program can proofread and the size of droplet image can change by zoom magnitude of CCD camcorder. However, actual size of the droplet depends on both hydrophobicity of sample and surface free energy of water that forms the droplet. Hence, droplet size also depends on the temperature and spraying condition.

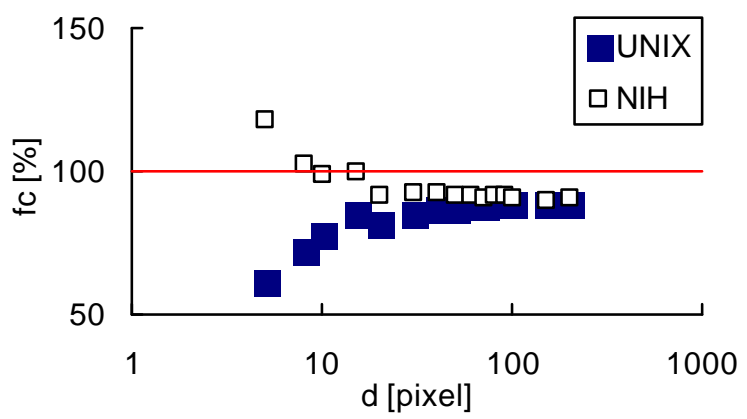

Figure 4: Digitizing error of the evaluation of fc. Circles of diameter, d, were made by MS Paint software.

UNIX: Evaluated from PVM program.

NIH: Evaluated from NIH Image.
Table 3: Evaluated circle image made by MS Paint and its calculated image indexes. Conditions: Diameter of the circle; $d=5$. UNIX; calculated by PVM program. NIH; measured by NIH Image by NIH (USA). Analog shows theoretical values of the circle with diameter $=5$.

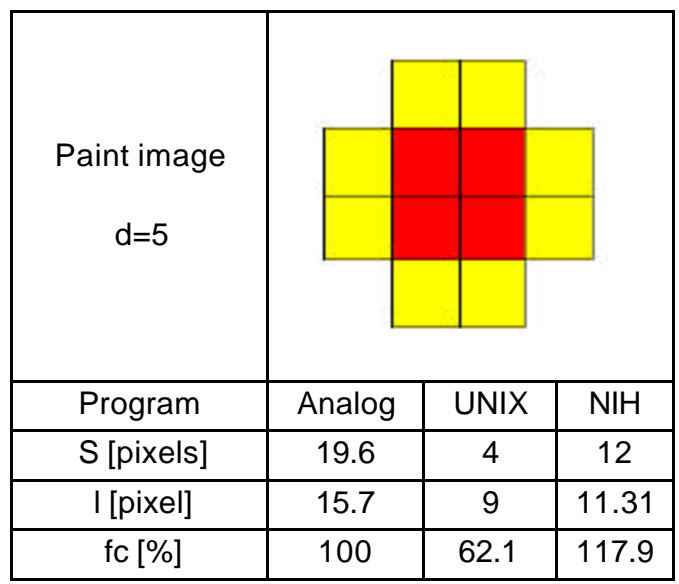

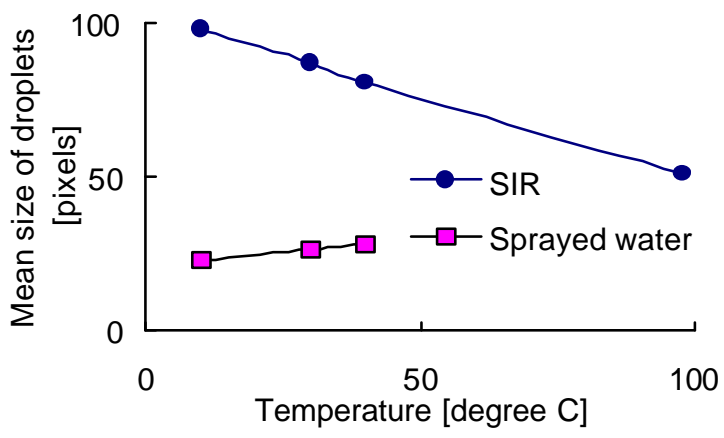

Figure 5: Temperature dependence of mean size of the droplets. SIR: Change the SIR temperature. Sprayed water is at room temperature. Sprayed water: Change the one of sprayed water. SIR is at room temperature.

\section{Temperature Dependence of Mean Size of the Water Droplets on SIR}

Figure 5 shows temperature dependence of mean size of droplets in images of SIR. •; SIR shows the mean size of water droplets when the temperature of SIR is changed. -; Sprayed water shows the one when the temperature of sprayed water is changed. The spraying and image digitizing conditions are same except the temperature of either SIR or Sprayed water.

Increasing the temperature of SIR is decreasing the surface free energy of SIR surface. It causes the sample hydrophobic and, therefore, droplets become smaller. Increasing the temperature of sprayed water also decreases the surface free energy of water. It increases the size of mist and, therefore, increases the mean size of water droplets on SIR. 


\section{Time Variation of fc during Spraying}

Figure 6 shows time variation of fc during spraying of distilled water on SIR at room temperature. Frame rate of CCD camcorder is 30 FPS. Temperatures of sprayed water are 10, 30 and 40 degree. It shows the spraying is finished during $0.3 \mathrm{sec}$ and $\mathrm{fc}$ increases during the formation of the droplets. Shape factor, fc, almost independent to the temperature of sprayed water when the size of droplets are enough.

\section{Time Variation of Mean Size of Droplets at AC Field Application}

To measure the effect of AC field application on the shape of the droplets on SIR, a pair of inter-digital electrodes made from stainless steel is put on SIR surface. Both the electrode's width and interval are 4 $\mathrm{mm}$. Thickness of the finger electrode is $3 \mathrm{~mm}$. Frame rate of the image is 30 FPS and applied field frequency is $29 \mathrm{~Hz}$.

Figure 7 shows the time variation of mean size of the droplets on SIR at low and high AC field application. If the deformation of droplets corresponds to the instantaneous ac field, mean size of droplets shows the sinusoidal response. However, Fig. 7 only shows the increase of its mean size even the ac field is high. This means the mean size of water droplets is increased by ac high field application and it does not decrease when the instantaneous AC field becomes low.

This phenomenon is also detected by the change in dielectric property measured by same electrode on the SIR surface [4]. Electric field dependence of dielectric properties, such as ac loss factor and the change in capacitance, have hysteresis when the residual contact angle becomes smaller.

When the sample surface has excellent hydrophobic property, its residual contact angle becomes larger. In this case, water droplets on the SIR show vibration during the application of ac high field. This vibration phenomenon depends on the size of the droplets and on the magnitude of applied field.

\section{Acknowledgment}

This research is supported by Grant-in-Aid for Scientific Research (B) (2) of the Ministry of Education, Science, Sports and Culture of Japan and by the research foundation for the Electrotechnology of Chubu. We thank to Mr. Sawada and Mr. Katayama for their help of experiments.

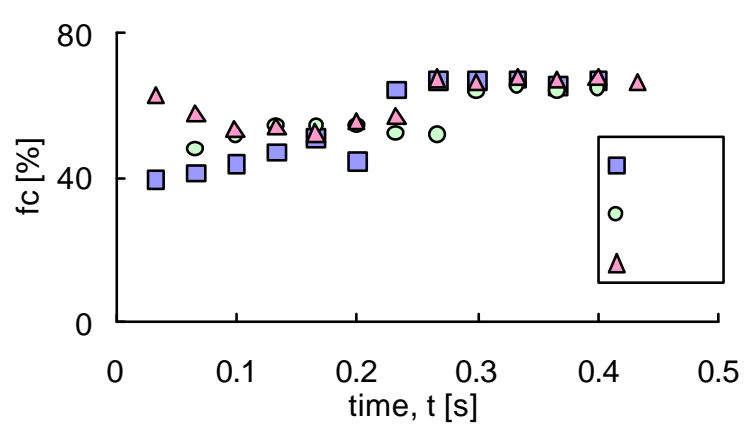

Figure 6: Time variation of fc of the droplets during spraying. Parameter: Temperature of sprayed water.

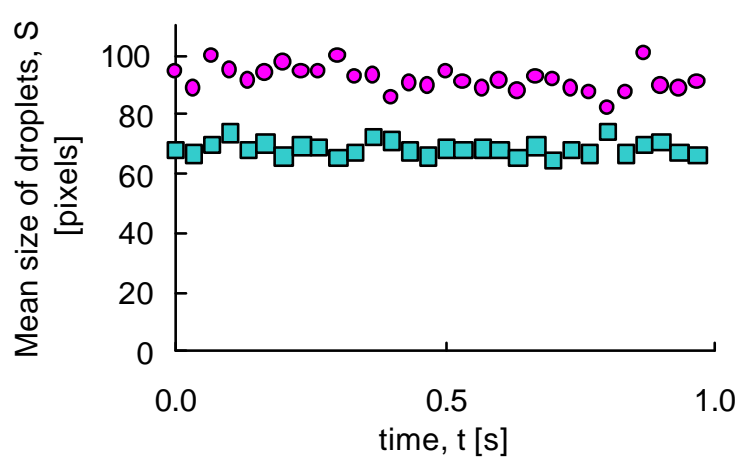

Figure 7: Time variation of mean size of the droplets on SIR. •: High field application. a: Low field application.

Image frame rate: 30 FPS. AC field frequency: $29 \mathrm{~Hz}$

\section{References}

[1] Swedish Transmission Research Institute: "Hydrophobicity Classification Guide", Guide 1, 92/1, 1992.

[2] Marcus Berg, Rajeev Thottappillil and Viktor Scuka, “A Digital Image Processing Method for Estimating the Level of Hydrophobicity of High Voltage Polymeric Insulating Materials", CEIDP, pp.756-762, 1999.

[3] T. Tokoro, M. Nagao and M. Kosaki, "Image Analysis of Hydrophobicity of Silicone Rubber Insulator", CEIDP, pp.763-766, 1999.

[4] H. Sindou T. Tokoro and M. Kosaki, "DSP Measurement of Dielectric Property of Polymeric Materials Using Interdigital Electrode", CEIDP, pp. 600-603, 2000

Author address: Tetsuro Tokoro, Department of Electrical and Computer Engineering, Gifu National College of Technology, Sinsei-cho, Motosu-gun, Gifu 501-0495 Japan, E-mail tokoro @ gifu-nct.ac.jp. 